\title{
Hybridization of Harmonic Search Algorithm In Training Radial Basis Function With Dynamic Decay Adjustment For Condition Monitoring
}

Hue Yee CHONG

University of Malaya: Universiti Malaya

Shing Chiang TAN

Multimedia University - Melaka Campus: Multimedia University

Hwa Jen Yap ( $\sim$ hjyap737@um.edu.my )

University of Malaya https://orcid.org/0000-0001-7637-9297

\section{Research Article}

Keywords: Metaheuristic, Radial Basis Function Network, condition monitoring, Harmony Search, Optimization

Posted Date: April 30th, 2021

DOI: https://doi.org/10.21203/rs.3.rs-174329/v1

License: (c) (i) This work is licensed under a Creative Commons Attribution 4.0 International License. Read Full License 


\title{
HYBRIDIZATION OF HARMONIC SEARCH ALGORITHM IN TRAINING RADIAL BASIS FUNCTION WITH DYNAMIC DECAY ADJUSTMENT FOR CONDITION MONITORING
}

\author{
Hue Yee CHONG ${ }^{1}$, Shing Chiang TAN ${ }^{2}$, and Hwa Jen YAP ${ }^{1 *}$ \\ ( ${ }^{\text {DDepartment }}$ of Mechanical Engineering, Faculty of Engineering, University Malaya, Kuala Lumpur 50603, \\ Malaysia \\ ${ }^{2}$ Faculty of Information Science \& Technology, Multimedia University, Melaka 75450, Malaysia)
}

\author{
*Corresponding author \\ Name: Hwa Jen YAP \\ Tel: +603-7967 5240 (Office) \\ Fax: +603-7957 5317 \\ Email address: hjyap737@um.edu.my
}

\begin{abstract}
In recent decades, hybridization of superior attributes of few algorithms was proposed to aid in covering more areas of complex application as well as improves performance. This paper presents an intelligent system integrating a Radial Basis Function Network with Dynamic Decay Adjustment (RBFN-DDA) with a Harmony Search (HS) to perform condition monitoring in industrial processes. An effective condition monitoring can help reduce unexpected breakdown incidents and facilitate in maintenance. RBDN-DDA performs incremental learning wherein its structure expands by adding new hidden units to include new information. As such, its training can reach stability in a shorter time compared to the gradient-descent based methods. By integrating with the HS algorithm, the proposed metaheuristic neural network (RBFN-DDA-HS) can optimize the RBFN-DDA parameters and improve classification performances from the original RBFN-DDA by $2.2 \%$ up to $22.5 \%$ in two benchmarks and a real-world condition-monitoring case studies. The results also show that the proposed RBFNDDA-HS is compatible, if not better than, the classification performances of other state-of-art machine learning methods.
\end{abstract}

Keywords: Metaheuristic, Radial Basis Function Network, condition monitoring, Harmony Search, Optimization

\section{Declarations:}

Funding: The authors would like to acknowledge the Ministry of Higher Education of Malaysia for the financial support under the Fundamental Research Grant Scheme (FRGS), Grant No: FP061-2015A and also the Ministry of Education Malaysia for the financial support under the program myBrain15.

Conflict of Interest: The authors declare that there is no conflict of interests regarding the publication of this paper.

Availability of data and material: NA

Code availability: NA

Authors' contributions: NA 


\section{Introduction}

Maintenance involves carrying out all technical and associated administrative activities to keep all components in an operational system to perform their function properly [1]. If any equipment breakdown occurs in a wellmaintained operational system, minor problems can be detected and corrected by conducting short daily inspection, cleaning and lubricating activities. An effective maintenance action requires company-wide participation and support by every personnel in order to make plant and equipment more reliable [2].

Basically, maintenance can be divided into two types, preventive maintenance and corrective maintenance [3]. Preventive maintenance includes all planned maintenance actions e.g. periodic inspection, condition monitoring etc. that are implemented to avoid equipment from breaking down unexpectedly. Corrective maintenance includes all planned and unplanned maintenance actions to rectify failures before restoring equipment back to its operational condition. Condition monitoring is a major component in predictive maintenance which monitors the condition and the significant changes of the machinery parameter to perform early detection and eliminate equipment defects that could cause unplanned downtime or incur unnecessary expenditures [4]. It is essential to develop efficient condition monitoring techniques for (1) quickly identifying the faulty components before breakdown. (2) reducing costly repairs caused by unexpected failure. (3) optimizing the scheduling of preventive and predictive maintenance operations without routine inspections, which may require periodic shutdowns in a plant. Condition monitoring consists of two sequential processes: feature extraction and condition classification [5]. Feature extraction requires the use of signal processing and/or post processing techniques; it is a mapping process from the signal space to the feature space. The condition classification is a process of classifying the obtained features into different categories. The traditional approach of condition monitoring relies on human expertise to relate the extracted features to the faults, which is usually time-consuming and unreliable, particularly when multiple features are referred for fault diagnostics and when the data are affected by noises [6]. Fault diagnosis coupled with artificial intelligence (AI) can potentially overcome the shortcoming of traditional signal processing techniques by incorporating the human-like thought abilities such as learning, reasoning and selfcorrection [7]. The widely used AI tools in condition monitoring include artificial neural network (ANN), fuzzy logic system, support vector machine, extreme learning machine and etc. [8]. Literature shows that ANNs with learning capabilities are useful models for tackling condition based maintenance problems [9]. They learn from data samples without a requirement for building an exact mathematical model. However, the performance of ANNs is highly depended on their parameter settings. For this concern, numerous global optimization methods are introduced and utilized to train ANNs to achieve a better network performance. Many of these global optimization methods are metaheuristic algorithms, which initiate a search process to explore in the search space to obtain near optimal solutions. The metaheuristic algorithms are mechanisms that imitate certain strategies inspired from nature, social behavior, physical laws, etc. Literature showed that many metaheuristic algorithms have been successfully used to optimize the ANN's design and the parameters [10]. Many reviews on ANN based condition monitoring have been presented [11, 12]. Notably, the application of evolutionary ANNs to condition monitoring is still relatively few. An effective evolutionary ANN-based condition monitoring model is helpful to reduce the frequency of unexpected breakdown incidents and thus, facilitate in maintenance. Thus, this motivates a research on developing an autonomous learning model based on the hybridization of an adaptive ANN and a metaheuristic algorithm for handling condition classification tasks in industries, such as in power systems. In this paper, a hybrid model of an incremental radial basis ANN and metaheuristic algorithm is developed. A metaheuristic algorithm (i.e., harmony search (HS) algorithm) is proposed to improve the learning parameters of the ANN. The research work is aimed at developing an evolutionary ANN for monitoring operating states of a system more accurately in a power plant.

After Section 1, the Section 2 of this paper will present a review of condition monitoring and fault diagnosis techniques. In addition, the state of art of ANN and evolutionary ANN models used for performing condition monitoring and fault detection will be described. The proposed evolutionary ANN model will be described in Section 3. The details of machine learning components (namely, Radial Basis Function Network with Dynamic Decay Adjustment (RBFN-DDA), Harmony Search (HS)) will be also presented before describing the proposed RBFN-DDA-HS in details. Section 4 reports and analyzes results in an experimental study involving condition 
monitoring on a circulating water system in a power plant. Finally, Section 5 draws concluding remarks and suggestions for further work.

\section{Related Work}

\subsection{Condition Monitoring and Fault Detection Techniques}

In general, condition monitoring and fault detection techniques can be broadly classified into model-based approach [13] and data-driven approach [14], which are shown in Figure 1. Many review articles are available to survey the application of techniques from data-driven and model-based approaches in process monitoring and fault detection [15-17]. The model-based approach involves a modeling process, which is based on fundamental understanding about the physics of the process. The model can be either of a qualitative or a quantitative type. Qualitative modeling [18] relies on the use of qualitative functions centered around different units in a process to express the relationship between the input and output of the system while quantitative modeling [19] is built by the use of mathematical function. The performance and reliability of the diagnostic system are assessed by the accuracy of the model. Thus, these approaches are usually applied only when an accurate analytical model is available. However, an accurate mathematical model is usually difficult to derive to represent complex mechanical systems, especially when the machines operate in uncertain and noisy environments [20]. Besides, model-based approaches also rely on human expertise to relate the extracted features to the faults, which is usually timeconsuming and unreliable, particularly when multiple features are utilized for fault diagnostics and when the data are affected by noises [6]. Data-driven approach overcomes the above mentioned limitation of the model-based approaches and is more viable to monitor industrial processes that are inherently automated. Data-driven approach requires for real data obtained from a data acquisition system to monitor or forecast the process behaviour of the system under monitoring. The commonly used data-driven methods can be divided into statistical [21] and AI approaches [8]. Methods under the statistical approach perform condition monitoring / fault detection by identifying the relationships among the data and these relationships are referred for making classification and prediction in the future. The commonly used statistical methods such as control chart, principle component analysis and partial least squares are easy to build and they can provide fast detection on abnormal situations [22]. To further improve the diagnosis performance, fault diagnosis coupled with AI with the human-like thought abilities such as learning, reasoning and self-correction [7] are extensively developed. Comprehensive reviews on the use of AI techniques for fault detection and condition monitoring on mechanical components such as induction machine [11] and planetary gearboxes [12] have been presented. The reviews show AI is useful to enhance the precision and accuracy of the condition monitoring / fault detection system. AI condition monitoring / fault detection systems have been developed by using, for examples, ANN, fuzzy logic system, support vector machine, extreme learning machine and etc. [8]. Among them, ANN is popular due to its capability of identifying the complex nonlinear relationships among features in a large dataset and hence, it can perform with an accurate prediction. 


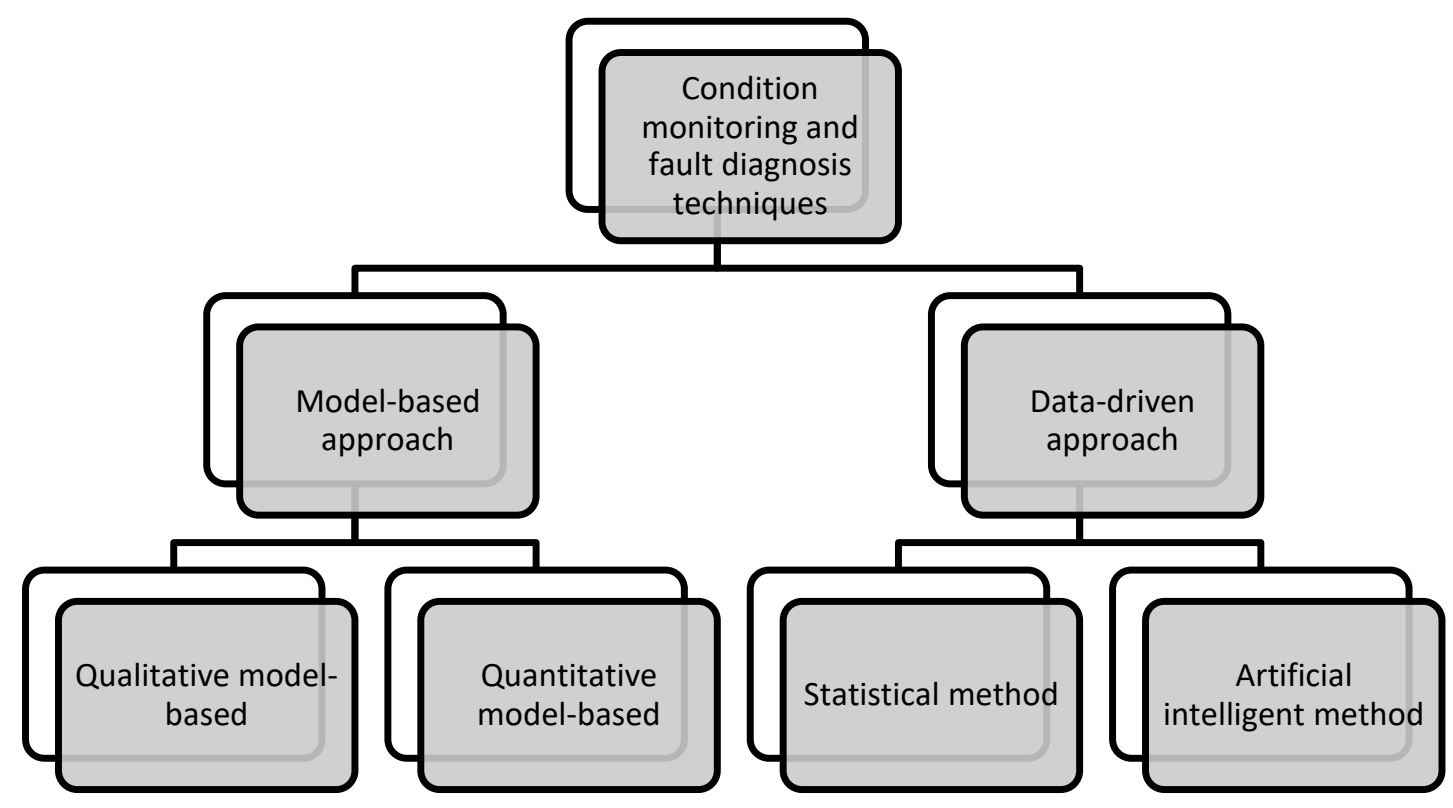

Figure 1: Overview of condition monitoring and fault detection approaches

\section{2 ANN and Evolutionary Neural Network Methods for Condition Monitoring and Fault Detection}

ANNs have been widely applied as condition classification tool in combination with signal processing techniques for feature extraction. For example, bearing has been identified as one of the main fault components in rotating machinery. From the literature, extensive research work on bearing fault detection and diagnosis have been reported [23]. A single hidden layer feed forward ANN was proposed to perform health diagnostics of ball bearings in direct-drive motors [24]. The feed forward ANN overcomes the problem of continuous change in rotational speed of the motor and identifies the health of ball bearing in different speed applications. A singular spectrum analysis (SSA) is integrated with an ANN based classifier to perform fault detection and diagnosis [25]. Feature extraction by using existing time series methods could be affected by noise and sample sizes, but the proposed method could overcome the drawback of the existing time series methods and achieve a high classification rate. A feature extraction method based on empirical mode decomposition (EMD) was appended to an ANN based classifier to categorize bearing defects [26]. The authors showed that the ANN can perform degradation assessment on the bearing condition automatically without human intervention. Kankar et.al compared the effectiveness of ANN and support vector machine (SVM) in detecting faults in a rotor bearing system [27]. They used statistical methods to extract features that were input to both ANN and SVM classifiers. Results showed that ANN classifier was able to achieve higher accuracy than SVM. An adaptive algorithm based on wavelet transform was applied to perform feature extraction and extracted features were input to ANN and KNN, where ANN showed more effective in classifying bearing faults [28]. Effective condition monitoring technique is useful to enhance a machining process. Siddhpura et.al presented a review of different classifiers (such as ANN, fuzzy logic, neurofuzzy, hidden markov model and SVM) used for predicting tool wear in metal cutting such [29]. Among them, ANN classifier was the most frequently used classifier due to its noise tolerant and high fault adaptability characteristics. A hybrid machine-learning classifier between Gaussian classifier and an adaptive resonance theory (ART) neural network was proposed to implement online tool wear monitoring [30]. This hybrid machine-learning classifier could memorize new knowledge in online training and achieve high classification accuracy. The performance of an ANN classifier was demonstrated to outperform SVM and KNN [31] in monitoring the condition of tool wear of a retrofitted CNC milling machine.

Although ANN has shown effective in various condition monitoring and fault detection applications, a drawback is that the performance of ANN is highly relied on the parameters in its architecture such as the number 
of hidden nodes and connection weights. A survey on different combination of ANNs and evolutionary algorithms (EAs) was first made by Yao [32]. Yao explained that connection weights, learning rules and architecture of ANNs could be evolved using EAs. After a decade, Azzini and Tettamanzi extended the Yao's survey by updating the most recent related literatures [33]. Ding et.al [34] reviewed and presented some of the problems associated to the integration of ANNs with EAs. In machine learning community, many metaheuristic algorithms have been introduced to integrate with ANNs to enhance learning for achieving a better generalization performance [35]. On the other hand, a number of comprehensive reviews on the application of ANNs in condition monitoring and fault detection are available in the literature; however, the work on integrating ANNs with metaheuristic algorithms for performing condition monitoring and fault detection is relatively few. Genetic algorithm (GA) is one of the popular evolutionary algorithms applied to perform fault detection. A Dual GA loop has been applied to optimize the structure and the connection weights of a three-layered feed-forward ANN for fault detection in power systems [36]. Besides, GA has been used together with Levenberg-Marquardt (LM) algorithm to train an ANN in an electrical machine fault detection application. The proposed hybrid method between GA and LM overcame the weal local search ability of GA [37]. Besides, the performance of various optimization methods including ANN, fuzzy, GA and Ant Colony Optimization (ACO) and their hybrid models are compared when predicting accidents caused by repair and maintenance in oil refineries [38]. Among those computing models, ANN-GA outperformed the rest. Three methods including multi-layer perceptron (MLP) neural network, radial basis function (RBF) neural network, and KNN were hybridized with GA for detecting gear faults [39]. Result showed that the performance of hybrid method was better than the single machine learning methods. Apart from evolutionary algorithm, swarm based metaheuristic algorithms are also always applied to train an ANN in fault detection applications. For example, particle swarm optimization (PSO) was used to hybridize with ANN in predicting drilling fluid density [40]. Result showed that PSO-ANN could achieve a better fault detection performance if compared to fuzzy inference system (FIS) and GA-FIS. PSO was also proposed to optimize the weights and threshold parameters of a back propagation neural network that was applied for drilling fluid density prediction [41]. In a fault detection of multilevel inverter, GA and PSO were applied separately to train an ANN in order to optimize its connection weights. PSO tended to predict more accurately and faster than GA [42]. On the other hand, four metaheuristic algorithms including PSO, GA, tabu search and cuckoo search have been applied to optimize the weight and the bias values of an ANN in a multilevel inverter fault diagnosis application [43]. A modified evolutionary PSO with time varying acceleration coefficient (MEPSO-TVAC) was proposed to optimize regression coefficient of the ANN before performing transformer fault detection [44]. Fault diagnosis performance could be enhanced on the use of a feature extraction method in addition to a decision making tool. Kernel linear discriminant analysis (KLDA) was proposed to extract the optimal features from a fault dataset of analog circuits, and PSO was applied to tune the ANN parameters and structures [45]. ACO was proposed to optimize the weights of an ANN in a rolling bearing fault detection application [46]. An improved Gravitational Search Algorithm (GSA) was to optimize the weights and the bias settings of an ANN trained by back propagation algorithm in machine vibration [47].

The performance of metaheuristic algorithm could be affected by the exploitation and exploration characteristics of the algorithm. Ideally, a metaheuristic algorithm should achieve a good balance of search between exploration and exploitation. One of the ways to tackle this issue is by hybridizing two or more metaheuristic algorithms in order to achieve such balance between two modes of search. In a rolling bearing fault detection and diagnosis, a wavelet packet decomposition (WPD) was utilized to extract data features, and a radical basis function neural network (RBFNN) was used to learn solutions from these data features. A biogeographybased optimization (BBO) and differential evolution (DE) were combined to improve the solution quality and convergence speed of the RBFNN [48]. A novel fault diagnosis model for sensor nodes was proposed by optimizing the weights of a feed forward ANN using a hybrid metaheuristic algorithm of GSA and PSO [49].

\section{Methods}

\subsection{RBFD-DDA}


Learning in RBFs is governed by many parameter settings such as the number of nodes in the hidden layers, the type of activation functions, the center and width of a neuron. Normally, in a fixed architecture, the number of nodes in the hidden layer must be determined or fixed before training process begins. It is important to determine the optimized number of neuron since redundant hidden neuron can result in poor generalization and overlearning situation. On the hand, insufficient number of hidden neuron can result in inadequate of learning information from data [50]. To enhance the performance of the network by determining optimized number of hidden neuron, the Dynamic Decay Adjustment (DDA) algorithm is applied. Two unique characteristics of DDA algorithm are the constructive nature of probabilistic extension of restricted coulomb energy algorithm (P-RCE) [51] a and the independent adaptation of each prototype by referring to a decay factor. An area of conflict is defined by using two thresholds including positive thresholds $\left(\theta^{+}\right)$and negative thresholds $\left(\theta^{-}\right)$during training as shown in Figure 2 [52]. $\theta^{+}$determines the lower bound of activation value for the training patterns of correct class so that no new neuron is committed while $\theta^{-}$represents the upper bound of activation value for the neuron tolerating with neurons of conflicting class. Thus, the area of conflict refers to those sections where neither matching nor conflicting training patterns are allowed to reside. This algorithm constructs an RBFN structure dynamically during training process by an idea that new hidden neurons will only be inserted to the hidden layer when necessary.

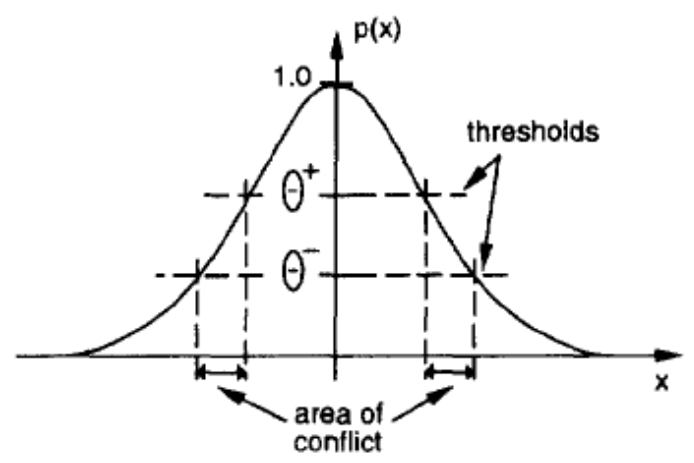

Figure 2: The DDA algorithm with two thresholds

With this growing structure during training process, to the RBFN can reach stability in a shorter time compared to ANNs trained with gradient-descent based methods. Besides, the DDA algorithm computes the decay factor based on neighbours' information. An RBFN trained with the DDA Algorithm (RBFN-DDA) consists of three layers, namely, the input, hidden and output layers (Figure 3). The input layer corresponding to network input features which represents the dimensionality of the input space and this layer is fully connected to the hidden layer. Next, the hidden layer of RBFN-DDA network consists of radial Gaussian units as an activation function and these units will only be added during training when necessary. Each hidden RBF unit is connected to only one output unit. The number of the output unit is determined by the number of the possible class. The output unit with the highest activation determines the class value. 


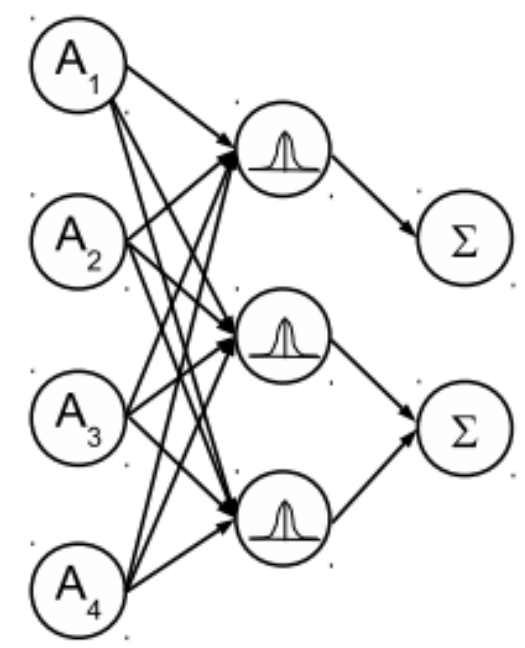

Figure 3: The structure of RBFN-DDA [53]

During the DDA-Algorithm training, all RBFs weights are first set to zero to avoid any duplication of the information about training samples. Then, all training samples are presented to the network. If a new training sample is classified correctly, then the weight of the RBF unit of correct class is increased by one. On the other hand, if the training sample is misclassified, a new RBF unit with an initial weight of one is introduced. The hidden RBF unit is assigned with a reference vector that is the same as the new training instance. The last step of the algorithm reduces the radii of all conflicting RBF units in a width shrinking process. The DDA algorithm defines an area of conflict by referring on two thresholds (positive thresholds $\left(\theta^{+}\right)$and negative thresholds $\left(\theta^{-}\right)$). In this study, the $\theta^{+}$and $\theta^{-}$are set to 0.4 and 0.2 . To commit a new RBF unit, the activation of existing RBFs of the correct class must not be above positive thresholds $\left(\theta^{+}\right)$and during shrinking the width of an RBF unit of a conflicting class must not be above negative thresholds $\left(\theta^{-}\right)$. Figure 4 shows the RBFN-DDA training in a single epoch. Usually, the training process of RBFN-DDA involves several epochs before completion. However, in this study, the center and radius of neuron shall be optimized using EA, thus the ANN training of RBFN-DDA is run for one epoch only.

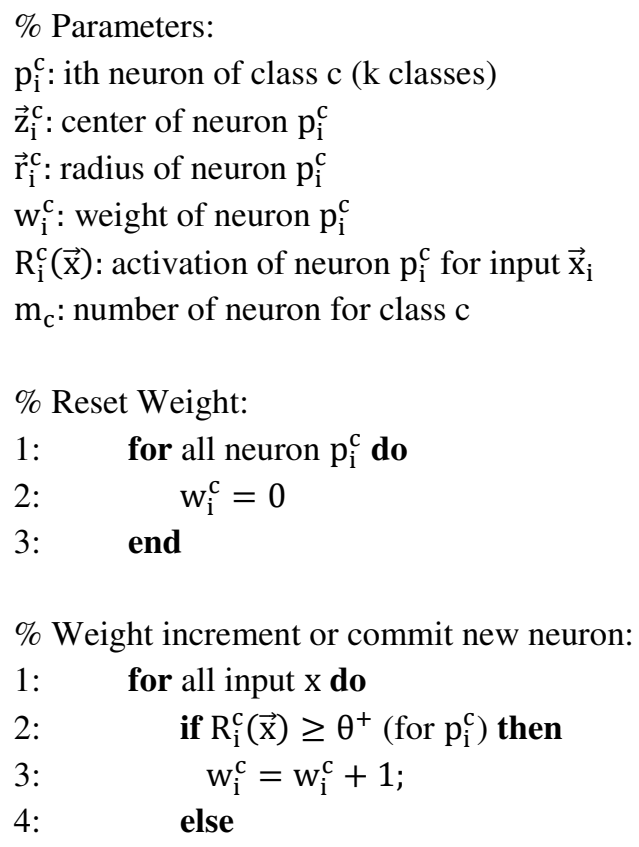




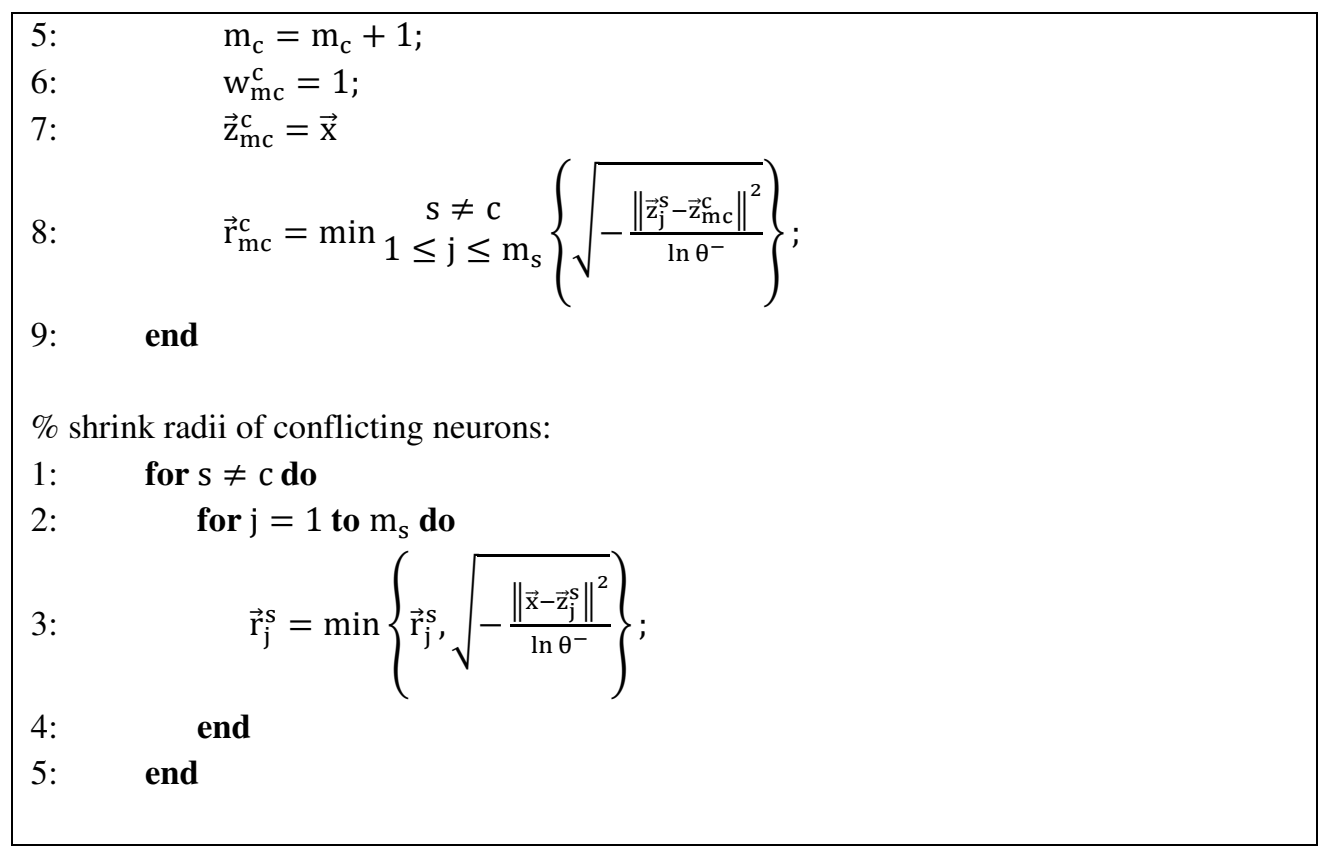

Figure 4: RBFN-DDA Training for one epoch

\subsection{Harmony Search $(H S)$}

An interesting musical inspired algorithm called HS algorithm was first introduced to solve optimization problems [54]. Musicians always search for an ideal state of harmony in their performances. Music improvisation is executed iteratively to obtain optimal harmony by considering three rules [54]: (1) memory consideration - a new music is improvised from the existing harmony memory (HM); (2) pitch adjustment - a new music is improvised by slightly adjusting the pitch; (3) randomization - a new music is improvised on a random basis. HS mimics a musician's behavior of searching for an ideal state of harmony for finding the best solution to an optimization problem. The ability to explore the search space of HS is highly depended on the pitch adjustment and randomization. Pitch adjustment warrants that new solution is improved from existing good solutions whereas randomization provides a search for new solution within the search space. Harmony memory size (HMS), harmony memory considering rate (HMCR), pitch adjusting rate (PAR), and the termination criterion shall be specified. The ability of HS in finding optimal solution from a search space is highly relied on a harmony memory acceptance rate. The following HS parameter ranges are recommended to produce an optimal solution [55] 0.70- 0.95 for HMCR, $0.20-0.50$ for PAR, and 10-50 for HMS. The procedure of HS algorithm is shown in Figure 5.

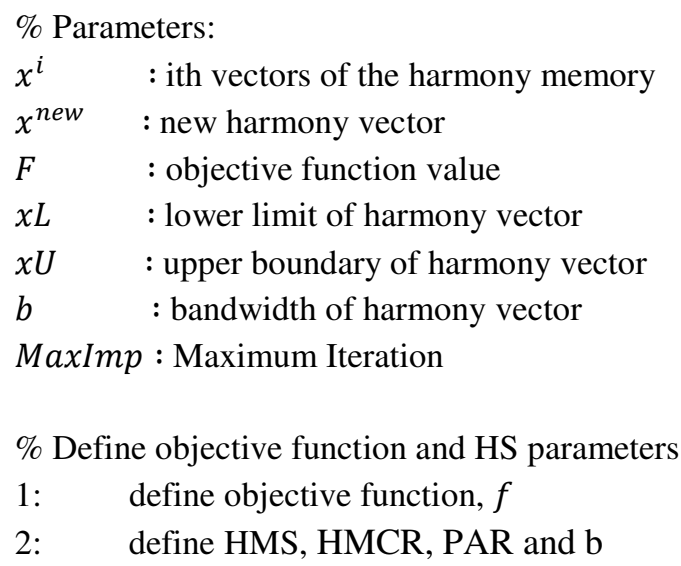




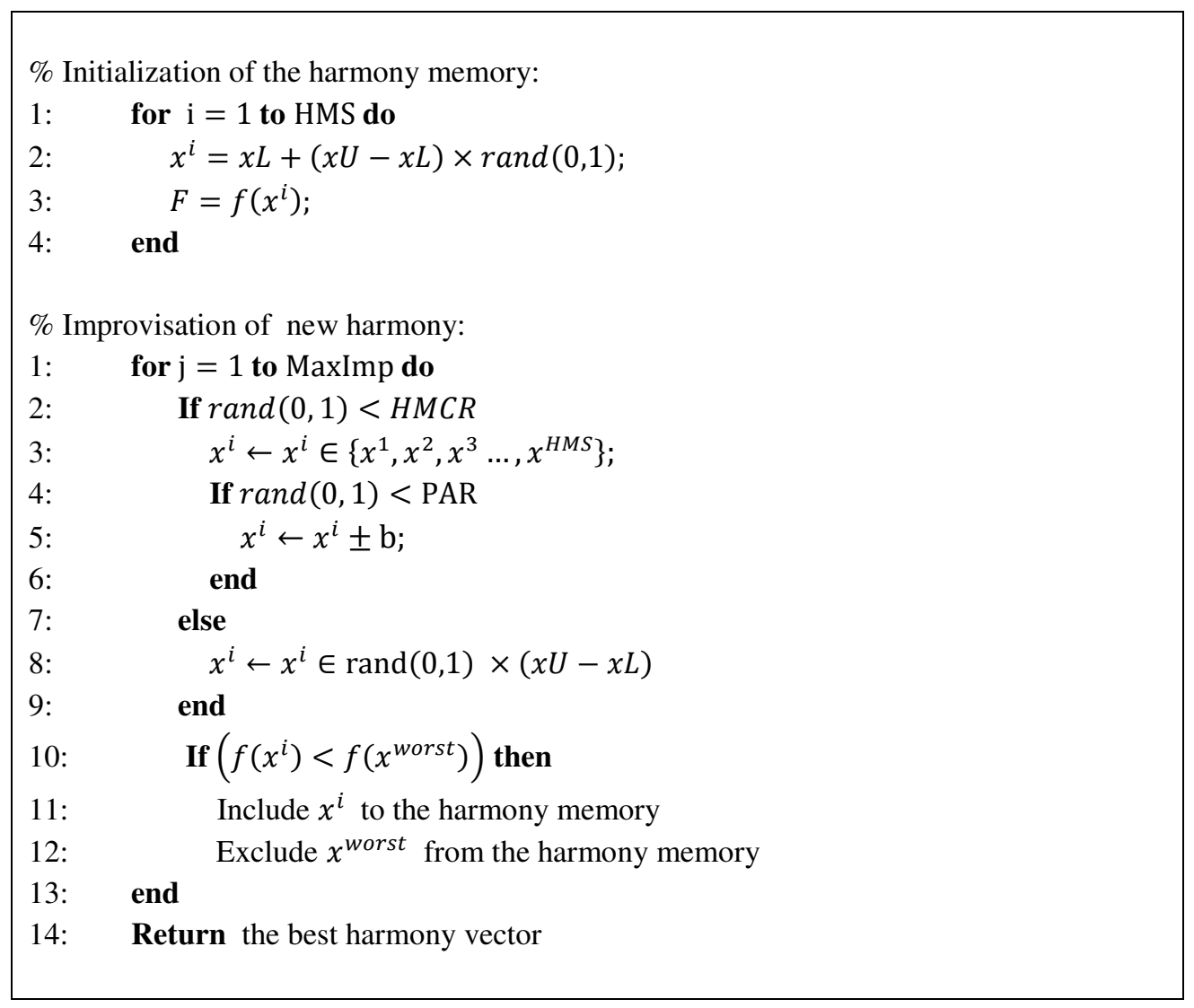

Figure 5: The HS algorithm procedure

\subsection{The Proposed Hybrid RBFN-DDA-HS}

In literature, many training algorithms were proposed to train RBFN, including gradient descent algorithm (GD) [56] and Kalman filtering (KF) [57]. However, these methods exhibit poor convergence and time consuming before obtaining optimal solution [58]. Evolutionary algorithm such as GA has been applied to optimize RBFN [59]. GA could perform a robust search algorithm to avoid itself from being stuck in local minima. However, its search algorithm is computational expensive to find optimal solution [60]. On the other hand, various global optimization algorithms based on other metaheuristics have been introduced to train RBFN for dealing with problems in different application domains. These global optimization algorithms include particle swarm optimization (PSO) algorithm [50], artificial immune system (AIS) algorithm [61], differential evolution (DE) algorithm [62], firefly algorithm (FA) [63]. The purpose of applying these algorithms is to find the best settings of the center and the width of the hidden units in RBFN to achieve optimal network performance [57]. In this paper, HS is proposed to optimize the center and the width of each hidden unit in a trained RBFN for optimizing its recognition performance. The reason why HS is adopted in this work is that conventional EA such as GA utilizes two parental vectors to generate new solution vectors whereas HS involves all existing vectors. Therefore, HS has a higher ability in obtaining better solutions when compared to those conventional EAs [64]. The training procedure of RBFN-DDA-HS model can be summarized as follows:

a. RBFN-DDA Training. The proposed model begins with the training of RBFN with the DDA Algorithm as described in Section 3.1 by using a training data set. After completing the RBFN-DDA training process, the trained solution vector is formed as a set of center and radius of hidden units $\left(\vec{z}^{\text {train }}, \vec{r}^{\text {train }}\right)$.

b. Defining objective function and setting the HS parameters. The fitness of the solution vector is evaluated in term of accuracy and hence the objective function is defined as below: 


$$
F\left(\vec{z}_{i}, \vec{r}_{i}\right)=\frac{C\left(\vec{z}_{i}, \vec{r}_{i}\right)}{N} \times 100
$$

where

$C\left(\vec{z}_{i}, \vec{r}_{i}\right)=$ The number of correctly classified training data by $\left(\vec{z}_{i}, \vec{r}_{i}\right)$

$\left(\vec{z}_{i}, \vec{r}_{i}\right)=$ The trained center and radius of a hidden node

$N=$ The number of training data

The HS parameter settings are listed in Table 1 below:

Table 1: The HS Parameter Settings

\begin{tabular}{lc}
\hline \multicolumn{1}{c}{ Harmony search parameter } & Value \\
\hline HMS & 10 \\
HMCR & 0.9 \\
PAR & 0.5 \\
Maximum Improvisation & 100 \\
\hline
\end{tabular}

Maximum improvisation is referred to as the largest number of iteration of the evolution in an HS. A common setting for maximum improvisation of HS is occasionally greater than 10,000 for achieving high accuracy rates. The reason is, the initial solution of HS is set randomly because prior knowledge about the problem is not known. A high setting in maximum improvisation is a way adopted by HS to search for solutions to achieve good accuracy rates. However, in this study, the initial harmony memory is not randomly set, it explores from a set of knowledge of the problem learned by the RBFN-DDA. RBFN-DDA that performs incremental learning can absorb information about the problem from the data and provides high quality initial solution. HS is employed to perform exploration on this solution with an aim to achieve high performance in terms of accuracy rates. Notably, the number of iteration of the HS component in RBFN-DDA-HS is set with a small number to avoid overtraining. Another advantage of RBFN-DDA-HS is that it can shorten computation time as compared to performing search using HS alone.

c. Forming an initial harmony memory from a trained RBFN. An initial population called harmony memory $(\mathrm{HM})$ is generated from a set of parameters from a trained RBFN instead of generating this population randomly. By referring Section 3.2, the HMS is set as 10. Thus, 10 sets of solution vector stored in the HM matrix $(\tilde{\mu}, r)$ as shown below:

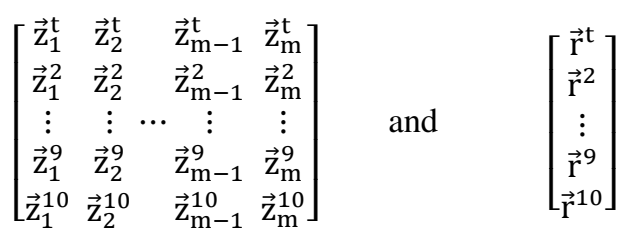

where $\left(\vec{z}^{t}, \vec{r}^{t}\right)$ is respectively the center and radius of RBFN; $m$ is the number of hidden units in RBFN. The other solution vectors $\left(\vec{z}^{i}, \vec{r}^{i}\right)$ are developed according to:

$$
\left(\vec{z}^{i}, \vec{r}^{i}\right)=\left(\vec{z}^{t}, \vec{r}^{t}\right) \pm \operatorname{rand}(0,1) \times R M F
$$

A Relative Multiple Factor $(\mathrm{RMF})$ value $\in[0,1]$ is applied on the trained solution vectors to control the variation of the developed centre and width. All solution vectors stored in the harmony memory are then evaluated by using the objective function defined in step (b). 
d. Evolving solution vectors. A new solution vector $\left(\vec{z}^{\text {new }}, \vec{r}^{\text {new }}\right)$ is generated either by slightly adjusting the solution candidate from any existing solution candidate in HM or by creating a new solution candidate on a random basis as described in Session 3.2 The fitness of new solution vector is computed as in step (b). If the new solution is better than the worst one, then it will be included in the HM while the worst one will be removed.

e. The procedure is either continued as in step (d) or is terminated if the maximum number of improvisations has been reached. A flow chart of the RBFN-DDA-HS model is listed in Figure 6.

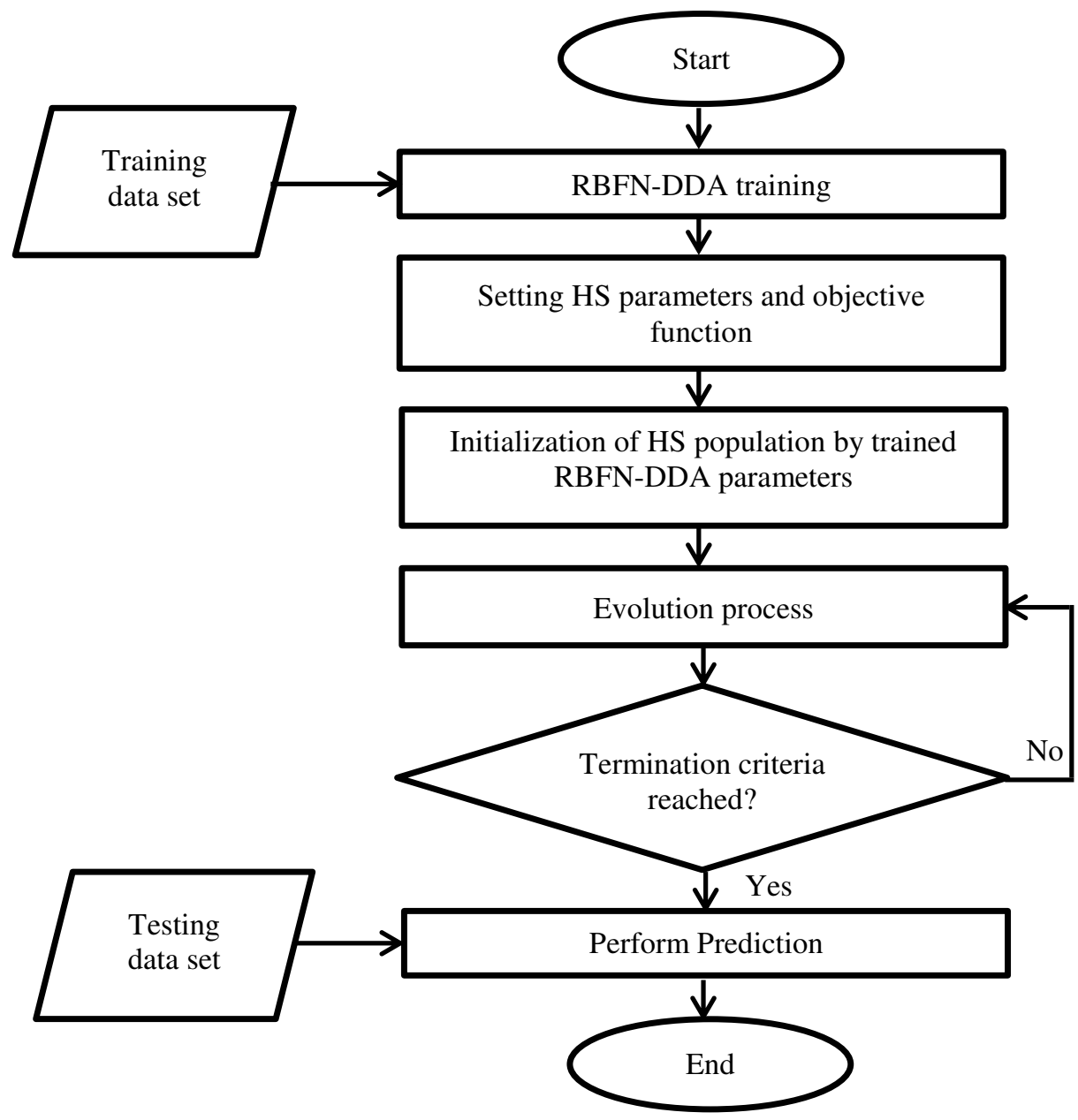

Figure 6: The flow chart of creating an RBFN-DDA-HS model

\section{Experiments and Results}

Fault detection is essential to ensure the effectiveness and reliability of machinery. In rotating machinery, bearing has been identified as one of the main fault components. Hence, fault detection of bearing has attracted a great attention from researchers. Besides, extensive research have been also focused on a data classification approach to fault detection in the manufacturing industry such as tool wear monitoring and machining parameter prediction that are described in Session 2.2. Condition monitoring and fault detection techniques are applied in power generation industry with an intention to avoid sudden breakdowns which may result in costly repair and machine unavailability. The effectiveness of the proposed model is evaluated in two benchmark fault detection problems, which are bearing fault classification and steel plate fault detection problems. This study also involves the application of the proposed RBFN model to perform condition monitoring in a real case study, which is a 
circulating water $(\mathrm{CW})$ system in a power generation plant. The dataset in each problem was randomly divided into both training and testing dataset. All the attributes in the dataset were normalized between 0 and 1 . To compare classification performances of RBFN-DDA and RBFN-DDA-HS statistically in monitoring the operating condition of the $\mathrm{CW}$ system, a Wilcoxon signed rank test was employed at a level of significance $\alpha=0.05$. The classification performance of the proposed RBFNDDA-HS was also compared with other machine learning methods in all three problems for which the results of these machine learning methods are taken from [20, 65-67] respectively.

\subsection{Fault Detection Applications}

\subsubsection{Benchmark dataset 1: Bearing data set}

The RBFD-DDA-HS is applied to classify the bearing faults. The benchmark dataset is taken from Case Western Reserve University (CWRU) [68]. The data were generated from a setup consisting of a $2 \mathrm{HP}$ motor, a dynamometer and bearing support (Figure 7). Data was collected from an accelerometer at $48000 \mathrm{~Hz}$ which means 48,000 samples / second was collected in the bearing experiments. The load was varied from 0 HP to 3 HP with fault dimensions varying from 0.1761 to $0.7044 \mathrm{~mm}$. Ten features were extracted from drive end signal, which were mean, standard deviation, variance, root mean square value, skewness, kurtosis, minimum value, peak value, crest factor and form factor. The bearing dataset contains 55 records that indicate four types of bearing fault condition including health bearing, inner race (IR) defect, ball defect and outer race (OR) defect. Details of this dataset are explained by Vakharia et al. [69].

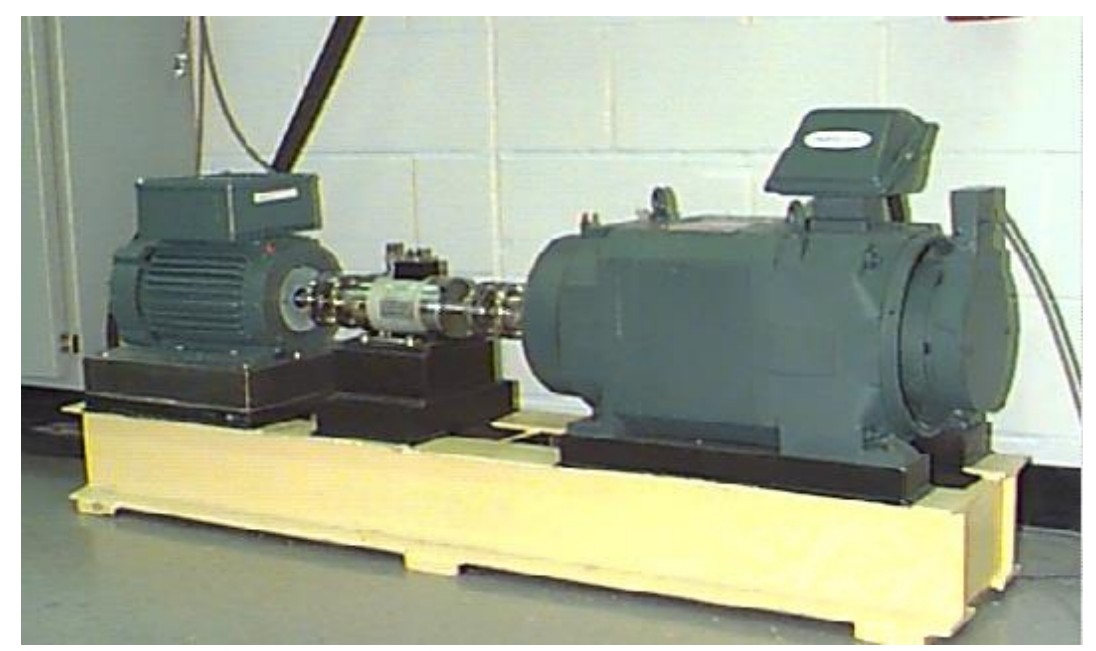

Figure 7: Bearing experiment setup [68]

\subsubsection{Benchmark dataset 2: Steel plate data set}

The data set for steel plate fault detection problem can be downloaded from the UCI web repository [70]. A total of 27 numerical attributes of a steel-plate image are used to classify each sample into one of the seven types of steel plate's faults, namely pastry, Z-scratch, K-scratch, stains, dirtiness, bumps, and others. The experiment is repeated for 30 times, with different training data to obtain average results. The data set consists of 1941 records which are divided into a training set of 1457 samples and a test set of 484 samples in the study.

\subsubsection{Case Study - The CW System}


The proposed RBFN-DDA-HS is applied to perform condition monitoring by learning and classifying a set of real data collected from a CW system in a power generation plant in Penang, Malaysia. The CW system operates to provide cooling water continuously to the main turbine condenser to condense steam from the turbine exhaust [71]. The overall water steam cycle efficiency in power plant is highly relied on the operating condition of the CW system. Intelligent system such as RBFN-DDA-HS could be helpful to monitor the operating conditions of the $\mathrm{CW}$ system and reduce the frequency of unexpected breakdown. Figure 8 shows an overview of the CW system. The $\mathrm{CW}$ system includes all piping and equipment (such as condensers and drum strainer) between seawater intake and the outfall where water is returned to the sea. The description of the method of seawater processing and how it is transferred into the $\mathrm{CW}$ system can be referred in [72]. A targeted 80MW power generation is the fundamental environment to establish the database. For every 5 minutes, an input sample of 12 temperature and pressure measurements was collected at the inlet and outlet points of the condenser. The operating conditions of the $\mathrm{CW}$ system were identified in four classes, which are listed in Table 2. The database had a total number of 2500 input samples.

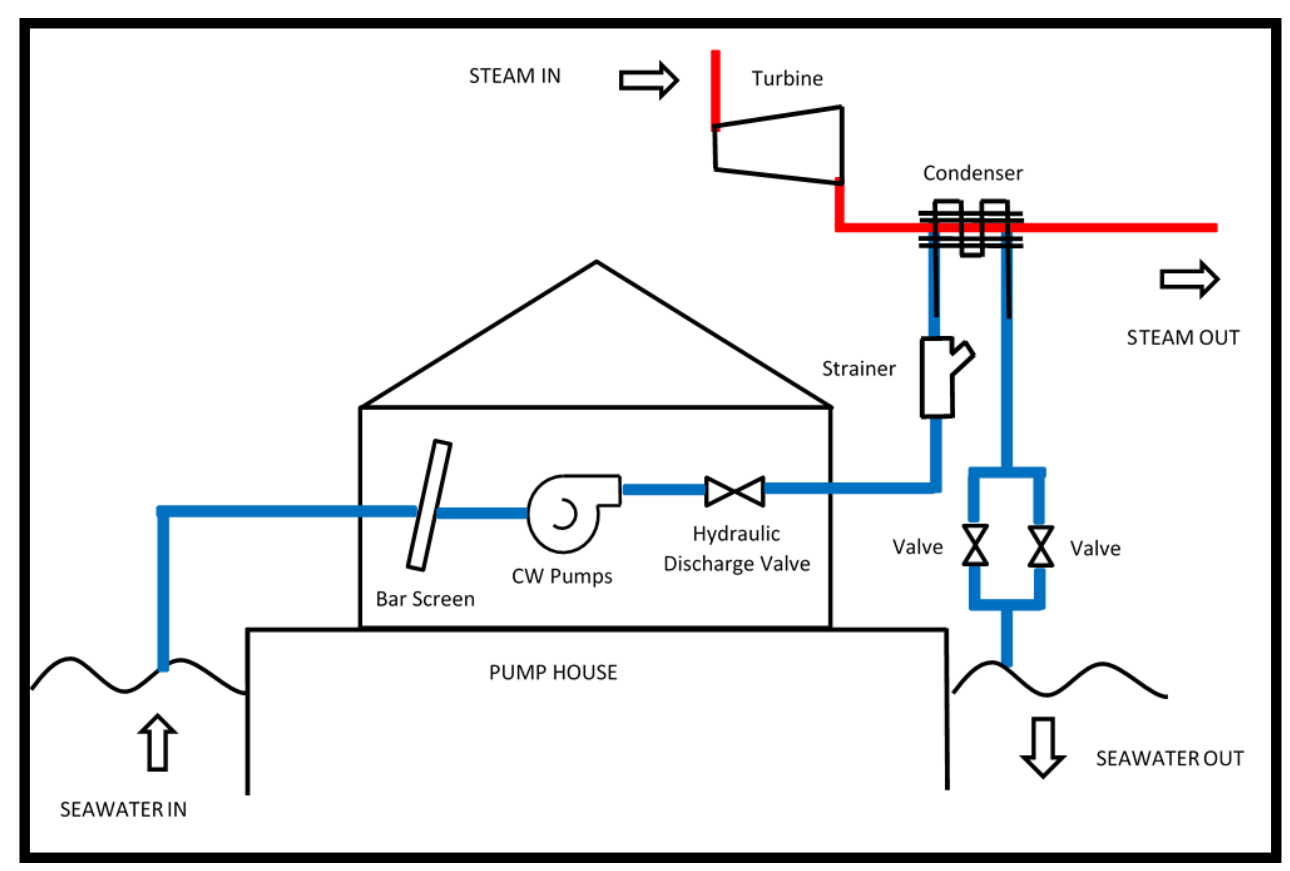

Figure 8: The circulating water (CW) system.

Table 2: Operating condition of the CW system

\begin{tabular}{cl}
\hline Class & \multicolumn{1}{c}{ Operation Condition } \\
\hline 1 & $\begin{array}{l}\text { Heat transfer in the condenser is efficient and there is no significant } \\
\text { blockage in the piping system } \\
\text { Heat transfer in the condenser is not efficient and there is no significant } \\
\text { blockage in the piping system }\end{array}$ \\
2 & $\begin{array}{l}\text { Heat transfer in the condenser is efficient and there is significant blockage } \\
\text { in the piping system }\end{array}$ \\
& $\begin{array}{l}\text { Heat transfer in the condenser is not efficient, and there is significant } \\
\text { blockage in the piping system }\end{array}$ \\
\hline
\end{tabular}


The experiment was conducted by repeatedly training the classifiers using different training and testing data before computing average classification results. We followed the experimental setup as mentioned in [65], [66] and [20]. In this case, for steel plate [66] problem and CW system case study [20], the experiment was repeated for thirty and ten times respectively. However, in experiment using the bearing dataset [65], the number of repetition of machine learning in training and testing were not mentioned. In our work, we repeat the experiment using RBFNDDA and RBFN-DDA-HS for thirty times. All average results of training and testing accuracy of RBFN-DDA and RBFN-DDA-HS are shown in Table 3. RBFN-DDA-HS performs with higher accuracy rates than RBFNDDA in all three dataset. These results signify the performance of RBFN-DDA has been improved after its learning is integrated with a search and adaptation process by the HS algorithm.

Table 3: Performance comparison between RBFN-DDA and RBFN-DDA-HS

\begin{tabular}{|c|c|c|c|c|c|c|}
\hline \multirow[b]{2}{*}{ Method } & \multicolumn{2}{|c|}{ (a) Bearing } & \multicolumn{2}{|c|}{ (b) Steel Plate } & \multicolumn{2}{|c|}{ (c) CW System Case Study } \\
\hline & $\begin{array}{c}\text { Training } \\
\text { Accuracy } \\
(\%)\end{array}$ & $\begin{array}{c}\text { Testing } \\
\text { Accuracy } \\
(\%)\end{array}$ & $\begin{array}{c}\text { Training } \\
\text { Accuracy } \\
(\%)\end{array}$ & $\begin{array}{c}\text { Testing } \\
\text { Accuracy } \\
(\%)\end{array}$ & $\begin{array}{c}\text { Training } \\
\text { Accuracy }(\%)\end{array}$ & $\begin{array}{c}\text { Testing } \\
\text { Accuracy } \\
(\%)\end{array}$ \\
\hline RBFN-DDA & 65.66 & 64.35 & 90.58 & 68.41 & 93.16 & 91.94 \\
\hline RBFN-DDA-HS & 80.06 & 78.81 & 92.59 & 69.94 & 96.56 & 96.20 \\
\hline
\end{tabular}

A Wilcoxon signed rank test [73] is applied to compare statistically the classification performance between RBFN-DDA and RBFN-DDA-HS at a level of significance $\alpha=0.05$ in all three problems. In this test, the null hypothesis is that the testing accuracy of RBFN-DDA-HS is the same as that of RBFN-DDA. The alternative hypothesis is that the testing accuracy of RBFN-DDA-HS is different from RBFN-DDA. By referring to the results in Table 4, all $p$-values are smaller than 0.05 . This means that the classification performances of RBFNDDA and RBFN-DDA-HS in terms of testing accuracy are statistically different. In this regard, RBFN-DDA-HS achieves better accuracy rates than RBFN-DDA in handling three tasks related to condition monitoring and fault detection.

Table 4: Results from the Wilcoxon rank tests (at the level of significance $\alpha=0.05$ )

\begin{tabular}{lcc}
\hline & Dataset & $\boldsymbol{p}$-value \\
\hline CW System & 0.002 \\
Bearing & $1.66 \times 10^{-6}$ \\
Steel Plate & $3.86 \times 10^{-6}$ \\
\hline
\end{tabular}

\subsubsection{Performance Comparison with Other Machine Learning Methods}

The classification performance of RBFN-DDA-HS is compared with other machine learning methods in all three problems [65], [66] and [20] from which their accuracy rates are referred. Table 4 shows the results. In bearing dataset, the result of RBFN-DDA-HS is compared with machine learning classifiers equipped with a feature selection algorithm (i.e., Random forest and Rotation Forest), and without a feature selection algorithm (i.e., ANN, SVM, Decision Tree, RBFN-DDA) [65]. The classification accuracy of RBFN-DDA (i.e. 64.35\%) is higher than the classifiers without feature selection such as ANN, SVM and Decision Tree, but is less accurate than classifiers with feature selection (i.e., Random forest and Rotation Forest). When RBFN-DDA classifier is integrated with HS, the proposed RBFN-DDA-HS model outperforms all other classifiers by achieving the highest classification accuracy, i.e., $78.81 \%$.

Next, in steel plate dataset the classification performance of RBFN-DDA-HS is compared with a metaheuristic classifier (FAM-GSA) and others single machine learning classifiers (FAM, MLP and RBF) [66]. Based on the results in Table 4, the testing accuracy of RBFN-DDA is higher than other single machine learning classifiers. Note that FAM-GSA contributes only a small improvement in testing accuracy, i.e., 0.9\% from FAM. Both FAM-GSA and RBFN-DDA-HS are meta-heuristic classifiers. In comparison, the proposed RBFN-DDA- 
HS is more effective than FAM-GSA in detecting steel plat faults. The HS can help improve RBFN-DDA-HS by a testing accuracy of $2.2 \%$ from RBFN-DDA.

The performance of the RBFN-DDA-HS in CW system is compared with fuzzy ARTMAP (FAM) rectangular basis function network (RecBFN), RBF-based Extreme Learning Machine and RBF-based Constrained Optimization Extreme Learning Machine (C-ELM). By observing the results in Table 5, the testing accuracy of RBFN-DDA is the lowest if compared to other machine learning methods. However, when RBFN-DDA is integrated with the HS algorithm to form the RBFN-DDA-HS, its testing accuracy improves greatly and outperforms all other machine learning methods. The HS algorithm is effective to search for the optimal parameter settings.

Table 5: Performance comparison in all three problems

\begin{tabular}{lclclc}
\hline \multicolumn{2}{c}{ (a) Bearing } & \multicolumn{2}{c}{ (b) Steel Plate } & \multicolumn{2}{c}{ (c) CW System Case Study } \\
\hline \multicolumn{1}{c}{ Model } & $\begin{array}{c}\text { Testing } \\
\text { Accuracy } \\
(\boldsymbol{\%})\end{array}$ & \multicolumn{1}{c}{ Model } & $\begin{array}{c}\text { Testing } \\
\text { Accuracy } \\
(\boldsymbol{\%})\end{array}$ & \multicolumn{1}{c}{ Model } & $\begin{array}{c}\text { Testing } \\
\text { Accuracy } \\
(\boldsymbol{\%})\end{array}$ \\
\hline ANN & 57.14 & FAM & 64.84 & FAM-RecBFN [20] & 95.70 \\
SVM & 46.42 & FAM-GSA & 65.40 & ELM (RBF) [67] & 94.80 \\
Decision Tree & 58.92 & MLP & 53.50 & C-ELM (RBF) [67] & 95.89 \\
Random forest & 66.07 & RBF & 59.94 & RBFN-DDA & 91.94 \\
Rotation Forest & 75.00 & RBFN-DDA & 68.41 & RBFN-DDA-HS & $\mathbf{9 6 . 2 0}$ \\
RBFN-DDA & 64.35 & RBFN-DDA-HS & $\mathbf{6 9 . 9 4}$ & & \\
RBFN-DDA-HS & $\mathbf{7 8 . 8 1}$ & & & & \\
\hline
\end{tabular}

\section{Summary}

In this study, a hybrid classification model of RBFN-DDA and the HS algorithm is proposed to improve the RBFN-DDA learning parameters. HS is adopted in the study due to its simplicity, high flexibility and search efficiency. The effectiveness of the proposed algorithm is demonstrated in the condition monitoring of circulating water $(\mathrm{CW})$ system and two fault detection benchmark datasets, which are bearing and steel plate. The proposed RBFN-DDA-HS achieves the highest classification accuracy if compared to other machine learning methods. Result shows RBFN-DDA-HS can improve classification performance in terms of testing accuracy that has a range between $2.2 \%$ and $22.5 \%$ from RBFN-DDA. Besides, the results from the Wilcoxon signed rank test show that the classification performances of RBFN-DDA and RBFN-DDA-HS are statistically different for all three condition-monitoring case studies where the latter outperforms the former. HS algorithm is a musical inspired metaheuristic algorithm. In the future, the classification performances of RBFN-DDA integrated with other types of metaheuristic algorithm (e.g., from biological, physical or chemical type) would be investigated. In addition, research may also focus on combining RBFN-DDA with two metaheuristic algorithms. The integration of two metaheuristic algorithm may explore the search space for optimal solutions more efficiently and effectively.

\section{Acknowledgement}

The authors would like to acknowledge the Ministry of Higher Education of Malaysia for the financial support under the Fundamental Research Grant Scheme (FRGS), Grant No: FP061-2015A and also the Ministry of Education Malaysia for the financial support under the program myBrain 15.

Conflict of Interest: The authors declare that there is no conflict of interests regarding the publication of this paper. 


\section{Reference}

1. Stephens, M.P., Productivity and Reliability-Based Maintenance Management. 2010: Purdue University Press.

2. Waeyenbergh, G. and L. Pintelon, A framework for maintenance concept development. International Journal of Production Economics, 2002. 77(3): p. 299-313.

3. Blanchard, B.S., Logistics Engineering and Management. 2004: Pearson Prentice Hall.

4. Mohanty, A.R., Machinery Condition Monitoring: Principles and Practices. 2014: CRC Press.

5. Marwala, T. and C.B. Vilakazi, Computational intelligence for condition monitoring. arXiv preprint arXiv:0705.2604, 2007.

6. $\quad$ Gertler, J., Fault Detection and Diagnosis in Engineering Systems. 1998: Taylor \& Francis.

7. Kok, J.N., et al., Artificial intelligence: definition, trends, techniques, and cases. Artificial intelligence, 2009. 1.

8. Ali, Y.H., Artificial Intelligence Application in Machine Condition Monitoring and Fault Diagnosis. Artificial Intelligence: Emerging Trends and Applications, 2018: p. 275.

9. Tallam, R.M., T.G. Habetler, and R.G. Harley, Stator winding turn-fault detection for closed-loop induction motor drives. Industry Applications, IEEE Transactions on, 2003. 39(3): p. 720-724.

10. Yao, X., A review of evolutionary artificial neural networks. International journal of intelligent systems, 1993. 8(4): p. 539-567.

11. Singh, G.K. and S.a. Ahmed Saleh Al Kazzaz, Induction machine drive condition monitoring and diagnostic research - a survey. Electric Power Systems Research, 2003. 64(2): p. 145-158.

12. Lei, Y., et al., Condition monitoring and fault diagnosis of planetary gearboxes: A review. Measurement, 2014. 48: p. 292-305.

13. Isermann, R., Supervision, fault-detection and fault-diagnosis methods - An introduction. Control Engineering Practice, 1997. 5(5): p. 639-652.

14. Patton, R.J., P.M. Frank, and R.N. Clark, Issues of fault diagnosis for dynamic systems. 2013: Springer Science \& Business Media.

15. Tidriri, K., et al., Bridging data-driven and model-based approaches for process fault diagnosis and health monitoring: A review of researches and future challenges. Annual Reviews in Control, 2016. 42 : p. 63-81.

16. Miljković, D. Fault detection methods: A literature survey. in 2011 Proceedings of the 34th International Convention MIPRO. 2011.

17. Ding, S.X., et al., A survey of the application of basic data-driven and model-based methods in process monitoring and fault diagnosis. IFAC Proceedings Volumes, 2011. 44(1): p. 12380-12388.

18. Venkatasubramanian, V., R. Rengaswamy, and S.N. Kavuri, A review of process fault detection and diagnosis. Computers \& Chemical Engineering, 2003. 27(3): p. 313-326.

19. Venkatasubramanian, V., et al., A review of process fault detection and diagnosis. Computers \& Chemical Engineering, 2003. 27(3): p. 293-311.

20. Tan, S.C., C.P. Lim, and M.V.C. Rao, A hybrid neural network model for rule generation and its application to process fault detection and diagnosis. Engineering Applications of Artificial Intelligence, 2007. 20(2): p. 203-213.

21. Yin, S., et al., A review on basic data-driven approaches for industrial process monitoring. IEEE Transactions on Industrial Electronics, 2014. 61(11): p. 6418-6428.

22. Venkatasubramanian, V., et al., A review of process fault detection and diagnosis. Computers \& Chemical Engineering, 2003. 27(3): p. 327-346.

23. Cerrada, M., et al., A review on data-driven fault severity assessment in rolling bearings. Mechanical Systems and Signal Processing, 2018. 99: p. 169-196.

24. Cocconcelli, M., et al. Diagnostics of ball bearings in varying-speed motors by means of Artificial Neural Networks. in Proceedings of the 8th International Conference on Condition Monitoring and Machinery Failure Prevention Technologies. 2011.

25. Muruganatham, B., et al., Roller element bearing fault diagnosis using singular spectrum analysis. Mechanical Systems and Signal Processing, 2013. 35(1-2): p. 150-166.

26. Ben Ali, J., et al., Application of empirical mode decomposition and artificial neural network for automatic bearing fault diagnosis based on vibration signals. Applied Acoustics, 2015. 89: p. 16-27.

27. Kankar, P.K., S.C. Sharma, and S.P. Harsha, Vibration-based fault diagnosis of a rotor bearing system using artificial neural network and support vector machine. International Journal of Modelling, Identification and Control, 2012. 15(3): p. 185-198. 
28. Gunerkar, R.S., A.K. Jalan, and S.U. Belgamwar, Fault diagnosis of rolling element bearing based on artificial neural network. Journal of Mechanical Science and Technology, 2019. 33(2): p. 505-511.

29. Siddhpura, A. and R. Paurobally, A review of flank wear prediction methods for tool condition monitoring in a turning process. The International Journal of Advanced Manufacturing Technology, 2013. 65(1-4): p. 371-393.

30. Wang, G., Y. Yang, and Z. Guo, Hybrid learning based Gaussian ARTMAP network for tool condition monitoring using selected force harmonic features. Sensors and Actuators A: Physical, 2013. 203: p. 394-404.

31. Hesser, D.F. and B. Markert, Tool wear monitoring of a retrofitted CNC milling machine using artificial neural networks. Manufacturing Letters, 2019. 19: p. 1-4.

32. Xin, Y., Evolving artificial neural networks. Proceedings of the IEEE, 1999. 87(9): p. 1423-1447.

33. Azzini, A. and A.G. Tettamanzi, Evolutionary ANNs: a state of the art survey. Intelligenza Artificiale, 2011. 5(1): p. 19-35.

34. Ding, S., et al., Evolutionary artificial neural networks: a review. Artificial Intelligence Review, 2013. 39(3): p. 251-260.

35. Devikanniga, D., K. Vetrivel, and N. Badrinath. Review of Meta-Heuristic Optimization based Artificial Neural Networks and its Applications. in Journal of Physics: Conference Series. 2019. IOP Publishing.

36. Bi, T.S., et al. A novel ANN fault diagnosis system for power systems using dual GA loops in ANN training. in 2000 Power Engineering Society Summer Meeting (Cat. No.00CH37134). 2000.

37. Zaiping, C., et al. Neural network electrical machine faults diagnosis based on multi-population GA. in 2008 IEEE International Joint Conference on Neural Networks (IEEE World Congress on Computational Intelligence). 2008.

38. Zaranezhad, A., H. Asilian Mahabadi, and M.R. Dehghani, Development of prediction models for repair and maintenance-related accidents at oil refineries using artificial neural network, fuzzy system, genetic algorithm, and ant colony optimization algorithm. Process Safety and Environmental Protection, 2019. 131: p. 331-348.

39. Lei, Y., et al., A multidimensional hybrid intelligent method for gear fault diagnosis. Expert Systems with Applications, 2010. 37(2): p. 1419-1430.

40. Ahmadi, M.A., et al., An accurate model to predict drilling fluid density at wellbore conditions. Egyptian Journal of Petroleum, 2018. 27(1): p. 1-10.

41. Zhou, H., et al. Effective calculation model of drilling fluids density and ESD for HTHP well while drilling. in IADC/SPE Asia Pacific Drilling Technology Conference. 2016. Society of Petroleum Engineers.

42. Sivakumar, M. and R. Parvathi, Application of Neural Network Trained with Metaheuristic Algorithms on Fault Diagnosis of Muti-level Inverter. Journal of Theoretical \& Applied Information Technology, 2014. 61(1).

43. Manjunath, T. and A. Kusagur, Analysis of Different Meta Heuristics Method in Intelligent Fault Detection of Multilevel Inverter with Photovoltaic Power Generation Source. International Journal of Power Electronics and Drive Systems, 2018. 9(3): p. 1214.

44. Illias, H.A., X.R. Chai, and A.H. Abu Bakar, Hybrid modified evolutionary particle swarm optimisationtime varying acceleration coefficient-artificial neural network for power transformer fault diagnosis. Measurement, 2016. 90: p. 94-102.

45. Xiao, Y. and L. Feng, A novel neural-network approach of analog fault diagnosis based on kernel discriminant analysis and particle swarm optimization. Applied Soft Computing, 2012. 12(2): p. 904920 .

46. Shi, H.W., ACO Trained ANN-Based Intelligent Fault Diagnosis. Applied Mechanics and Materials, 2010. 20-23: p. 141-146.

47. Do, Q.H., Predictions of Machine Vibrations Using Artificial Neural Networks Trained by Gravitational Search Algorithm and Back-Propagation Algorithm. Intelligence, 2017. 15(1): p. 93-111.

48. Zhang, Q., et al., WPD and DE/BBO-RBFNN for solution of rolling bearing fault diagnosis. Neurocomputing, 2018. 312: p. 27-33.

49. Khilar, P.M. and T. Dash, Multifault diagnosis in WSN using a hybrid metaheuristic trained neural network. Digital Communications and Networks, 2020. 6(1): p. 86-100.

50. Liu, Y., et al. Training radial basis function networks with particle swarms. in International Symposium on Neural Networks. 2004. Springer.

51. Hudak, M.J., Rce classifiers: Theory and practice. Cybernetics and System, 1992. 23(5): p. 483-515.

52. Berthold, M.R. and J. Diamond, Constructive training of probabilistic neural networks. Neurocomputing, 1998. 19(1): p. 167-183. 
53. Robnik-Šikonja, M., Data generators for learning systems based on RBF networks. IEEE transactions on neural networks and learning systems, 2016. 27(5): p. 926-938.

54. Geem, Z.W., J.H. Kim, and G. Loganathan, A new heuristic optimization algorithm: harmony search. Simulation, 2001. 76(2): p. 60-68.

55. Lee, K.S., et al., The harmony search heuristic algorithm for discrete structural optimization. Engineering Optimization, 2005. 37(7): p. 663-684.

56. Karayiannis, N.B., Reformulated radial basis neural networks trained by gradient descent. IEEE Transactions on Neural Networks, 1999. 10(3): p. 657-671.

57. Simon, D., Training radial basis neural networks with the extended Kalman filter. Neurocomputing, 2002. 48(1): p. 455-475.

58. Kurban, T. and E. Beşdok, A comparison of RBF neural network training algorithms for inertial sensor based terrain classification. Sensors, 2009. 9(8): p. 6312-6329.

59. Barreto, A.M., H.J. Barbosa, and N.F. Ebecken. Growing compact RBF networks using a genetic algorithm. in Neural Networks, 2002. SBRN 2002. Proceedings. VII Brazilian Symposium on. 2002. IEEE.

60. Hamadneh, N., et al., Learning logic programming in radial basis function network via genetic algorithm. Journal of Applied Sciences(Faisalabad), 2012. 12(9): p. 840-847.

61. de Castro, L.N. and F.J. Von Zuben. An immunological approach to initialize centers of radial basis function neural networks. in Proc. of CBRN'01 (Brazilian Conference on Neural Networks). 2001.

62. Yu, B. and X. He. Training radial basis function networks with differential evolution. in In Proceedings of IEEE International Conference on Granular Computing. 2006. Citeseer.

63. Horng, M.-H., et al., Firefly meta-heuristic algorithm for training the radial basis function network for data classification and disease diagnosis. 2012: INTECH Open Access Publisher.

64. Mahdavi, M., M. Fesanghary, and E. Damangir, An improved harmony search algorithm for solving optimization problems. Applied Mathematics and Computation, 2007. 188(2): p. 1567-1579.

65. Kavathekar, S., N. Upadhyay, and P. Kankar, Fault classification of ball bearing by rotation forest technique. Procedia Technology, 2016. 23: p. 187-192.

66. Tan, S.C. and C.P. Lim, Evolving an adaptive artificial neural network with a gravitational search algorithm, in Intelligent Decision Technologies. 2015, Springer. p. 599-609.

67. Wong, S.Y., Novel Extreme Learning Machines For Pattern Classification, Data Regression, And Rules Generation, in College Of Engineering2015, Universiti Tenaga Nasional: Malaysia.

68. Loparo, K.A., Bearing vibration data set.

69. Vakharia, V., V.K. Gupta, and P.K. Kankar, A comparison of feature ranking techniques for fault diagnosis of ball bearing. Soft Comput., 2016. 20(4): p. 1601-1619.

70. Lichman, M., UCI Machine Learning Repository, 2013, University of California, Irvine, School of Information and Computer Sciences.

71. Tenaga Nasional Berhad, System description and operating procedures, 1999: Prai Power Station Stage 3.

72. Tan, S.C. and C.P. Lim, Application of an adaptive neural network with symbolic rule extraction to fault detection and diagnosis in a power generation plant. IEEE Transactions on Energy Conversion, 2004. 19(2): p. 369-377.

73. Woolson, R., Wilcoxon signed-rank test. Wiley encyclopedia of clinical trials, 2007: p. 1-3. 
Figures

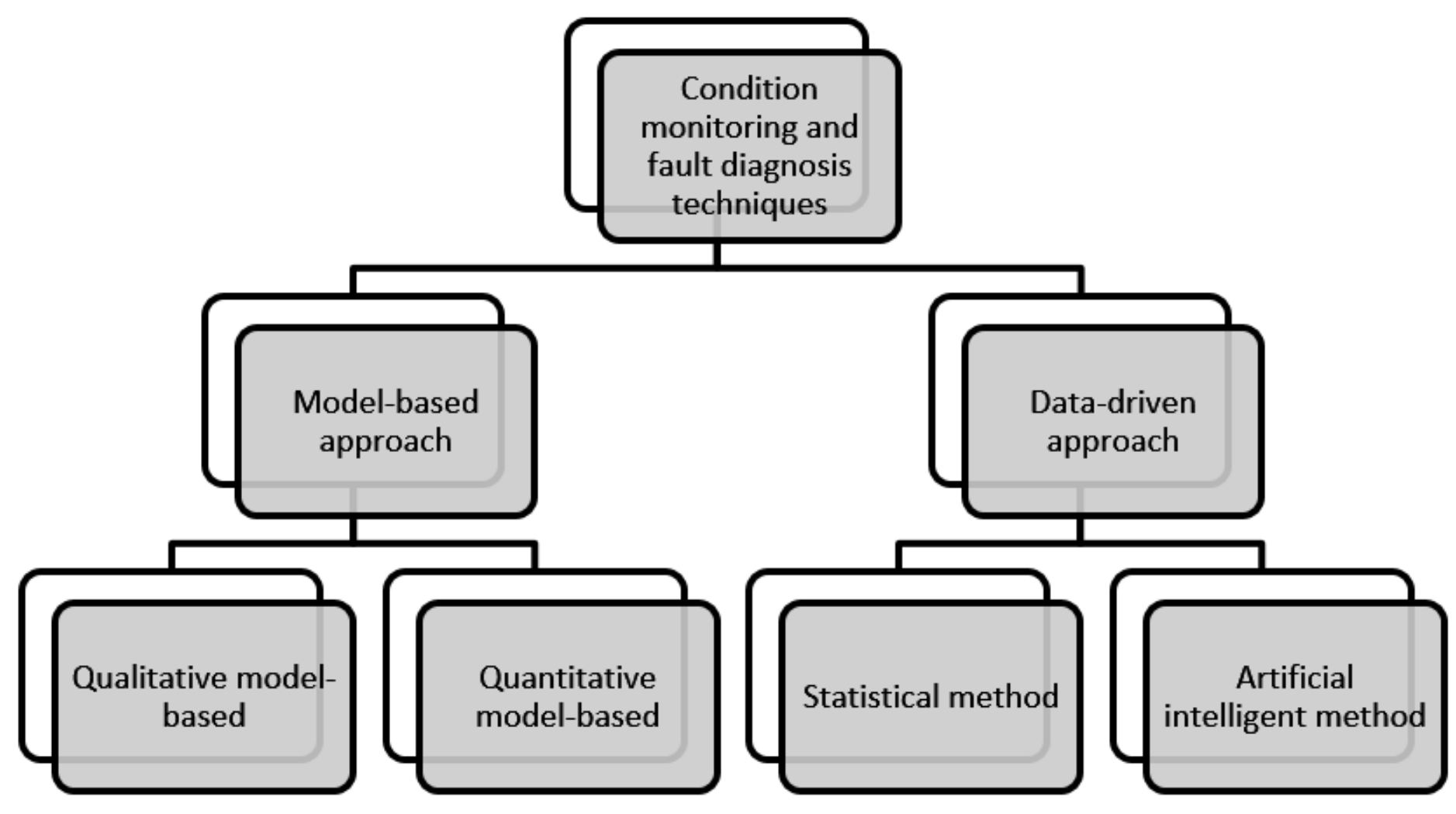

Figure 1

Overview of condition monitoring and fault detection approaches

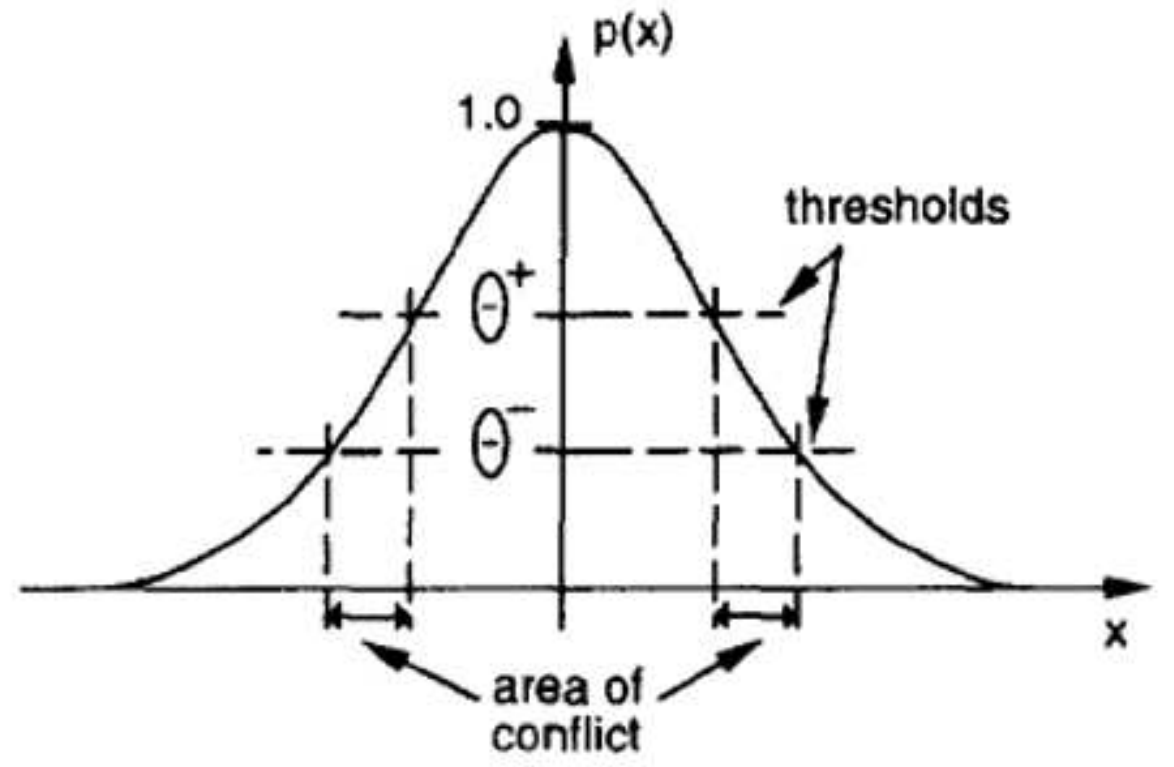

Figure 2 
The DDA algorithm with two thresholds

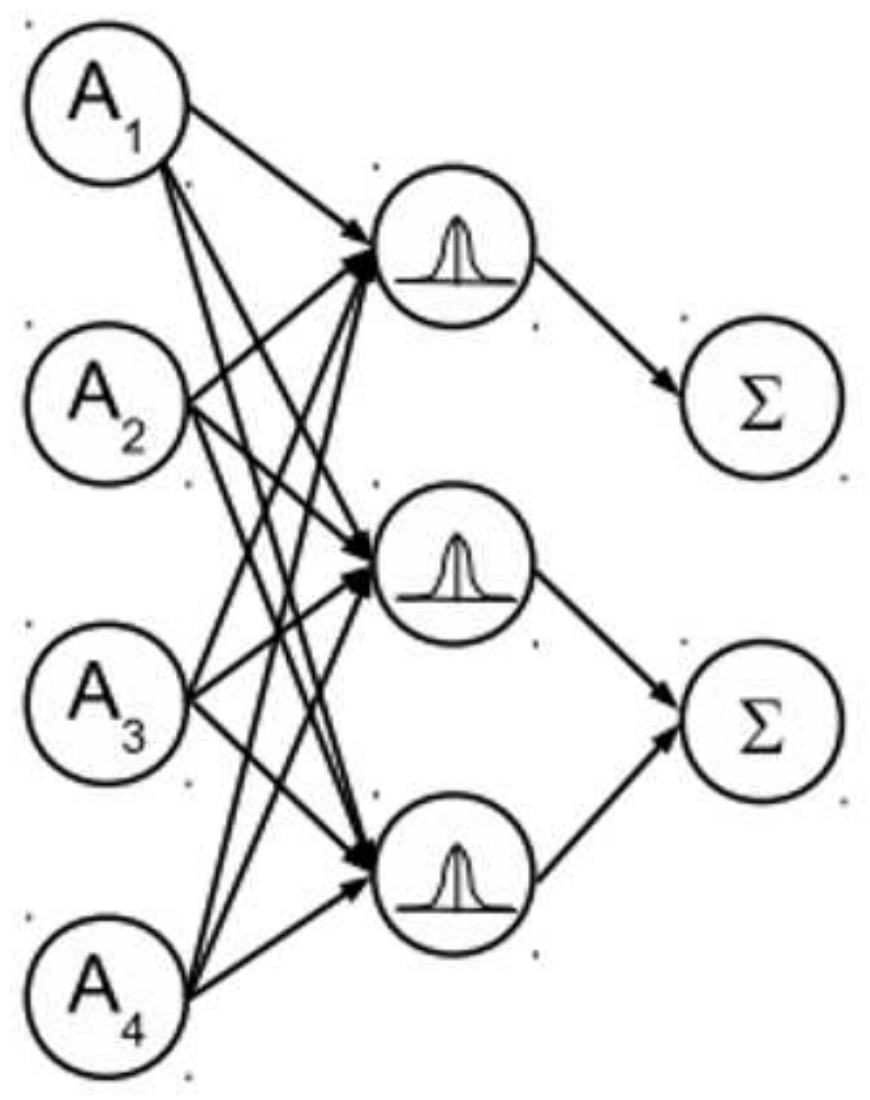

Figure 3

The structure of RBFN-DDA [53] 


\section{\% Parameters:}

$\mathrm{p}_{\mathrm{i}}^{\mathrm{c}}$ : ith neuron of class $\mathrm{c}$ ( $\mathrm{k}$ classes)

$\overrightarrow{\mathrm{z}}_{\mathrm{i}}^{\mathrm{c}}$ : center of neuron $\mathrm{p}_{\mathrm{i}}^{\mathrm{c}}$

$\vec{r}_{i}^{c}$ : radius of neuron $p_{i}^{c}$

$w_{i}^{c}$ : weight of neuron $p_{i}^{c}$

$R_{i}^{c}(\vec{x})$ : activation of neuron $p_{i}^{c}$ for input $\vec{x}_{i}$

$\mathrm{m}_{\mathrm{c}}$ : number of neuron for class $\mathrm{c}$

$\%$ Reset Weight:

1: for all neuron $\mathrm{p}_{\mathrm{i}}^{\mathrm{c}}$ do

2: $\quad \mathrm{w}_{\mathrm{i}}^{\mathrm{c}}=0$

3: $\quad$ end

$\%$ Weight increment or commit new neuron:

1: for all input $x$ do

2: $\quad$ if $R_{i}^{c}(\vec{x}) \geq \theta^{+}\left(\right.$for $\left.p_{i}^{c}\right)$ then

3: $\quad w_{i}^{c}=w_{i}^{c}+1$;

4: else

\begin{tabular}{|c|c|c|}
\hline 5: & $\mathrm{m}_{\mathrm{c}}=\mathrm{m}_{\mathrm{c}}+1 ;$ & \\
\hline 6: & $\mathrm{w}_{\mathrm{mc}}^{\mathrm{c}}=1$ & \\
\hline 7: & $\overrightarrow{\mathrm{z}}_{\mathrm{mc}}^{\mathrm{c}}=\overrightarrow{\mathrm{x}}$ & \\
\hline 8: & $\overrightarrow{\mathrm{r}}_{\mathrm{mc}}^{\mathrm{c}}=\min _{1 \leq \mathrm{j} \leq \mathrm{m}_{\mathrm{s}}}$ & $-\frac{\left\|\vec{z}_{j}^{s}-\vec{z}_{m c}^{c}\right\|^{2}}{\ln \theta^{-}}$ \\
\hline
\end{tabular}

9: $\quad$ end

$\%$ shrink radii of conflicting neurons:

1: $\quad$ for $\mathrm{s} \neq \mathrm{c}$ do

2: $\quad$ for $\mathrm{j}=1$ to $\mathrm{m}_{\mathrm{s}}$ do

3: $\quad \overrightarrow{\mathrm{r}}_{\mathrm{j}}^{\mathrm{s}}=\min \left\{\overrightarrow{\mathrm{r}}_{\mathrm{j}}^{\mathrm{s}}, \sqrt{-\frac{\left\|\overrightarrow{\mathrm{x}}-\overrightarrow{\mathrm{z}}_{\mathrm{j}}^{\mathrm{s}}\right\|^{2}}{\ln \theta^{-}}}\right\}$;

4: $\quad$ end

5: end

\section{Figure 4}

RBFN-DDA Training for one epoch 
$\%$ Parameters:

$x^{i} \quad:$ ith vectors of the harmony memory

$x^{\text {new }} \quad$ : new harmony vector

$F \quad$ : objective function value

$x L \quad$ : lower limit of harmony vector

$x U \quad$ : upper boundary of harmony vector

$b \quad$ : bandwidth of harmony vector

MaxImp : Maximum Iteration

$\%$ Define objective function and HS parameters

1: define objective function, $f$

2: $\quad$ define HMS, HMCR, PAR and b

$\%$ Initialization of the harmony memory:

1: for $\mathrm{i}=1$ to HMS do

2: $\quad x^{i}=x L+(x U-x L) \times \operatorname{rand}(0,1)$;

3: $\quad F=f\left(x^{i}\right)$;

4: $\quad$ end

\% Improvisation of new harmony:

1: $\quad$ for $\mathrm{j}=1$ to MaxImp do

2: $\quad$ If $\operatorname{rand}(0,1)<H M C R$

3: $\quad x^{i} \leftarrow x^{i} \in\left\{x^{1}, x^{2}, x^{3} \ldots, x^{H M S}\right\}$;

4: $\quad$ If $\operatorname{rand}(0,1)<$ PAR

5: $\quad x^{i} \leftarrow x^{i} \pm \mathrm{b}$;

6: $\quad$ end

7: $\quad$ else

8: $\quad x^{i} \leftarrow x^{i} \in \operatorname{rand}(0,1) \times(x U-x L)$

9: $\quad$ end

10: $\quad$ If $\left(f\left(x^{i}\right)<f\left(x^{\text {worst }}\right)\right)$ then

11: Include $x^{i}$ to the harmony memory

12: $\quad$ Exclude $x^{\text {worst }}$ from the harmony memory

13: end

14: Return the best harmony vector

\section{Figure 5}

The HS algorithm procedure 


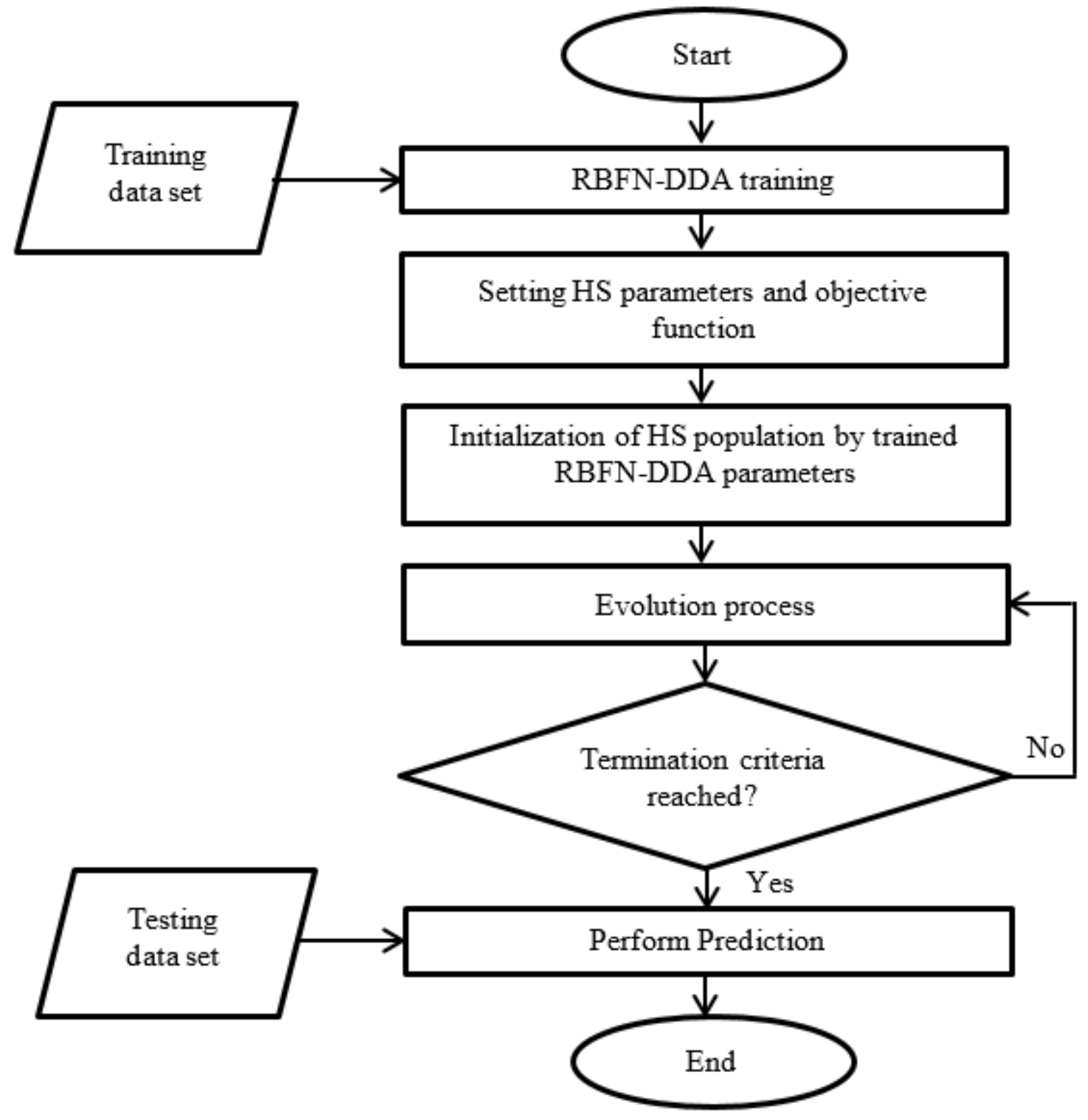

Figure 6

The flow chart of creating an RBFN-DDA-HS model 


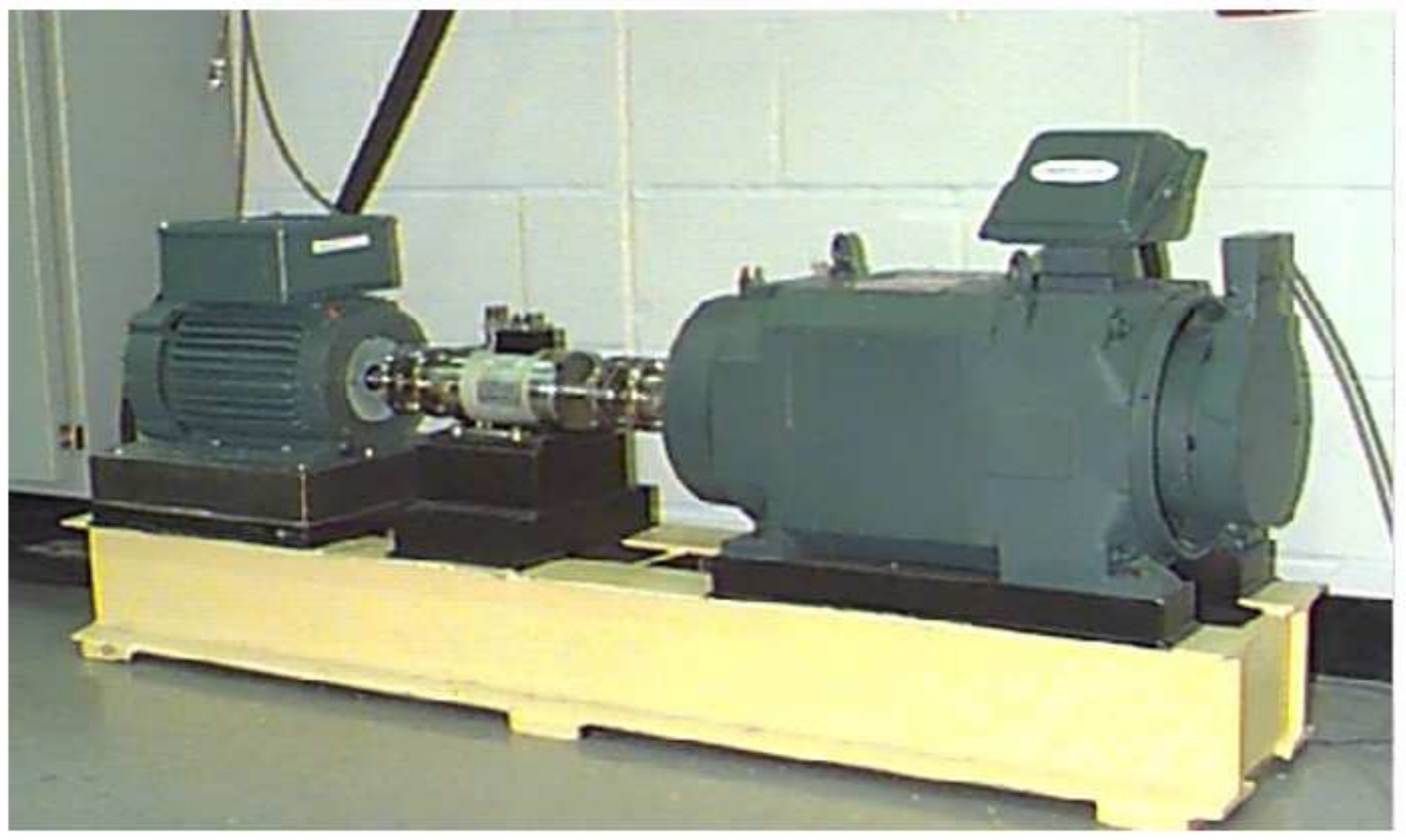

Figure 7

Bearing experiment setup [68] 


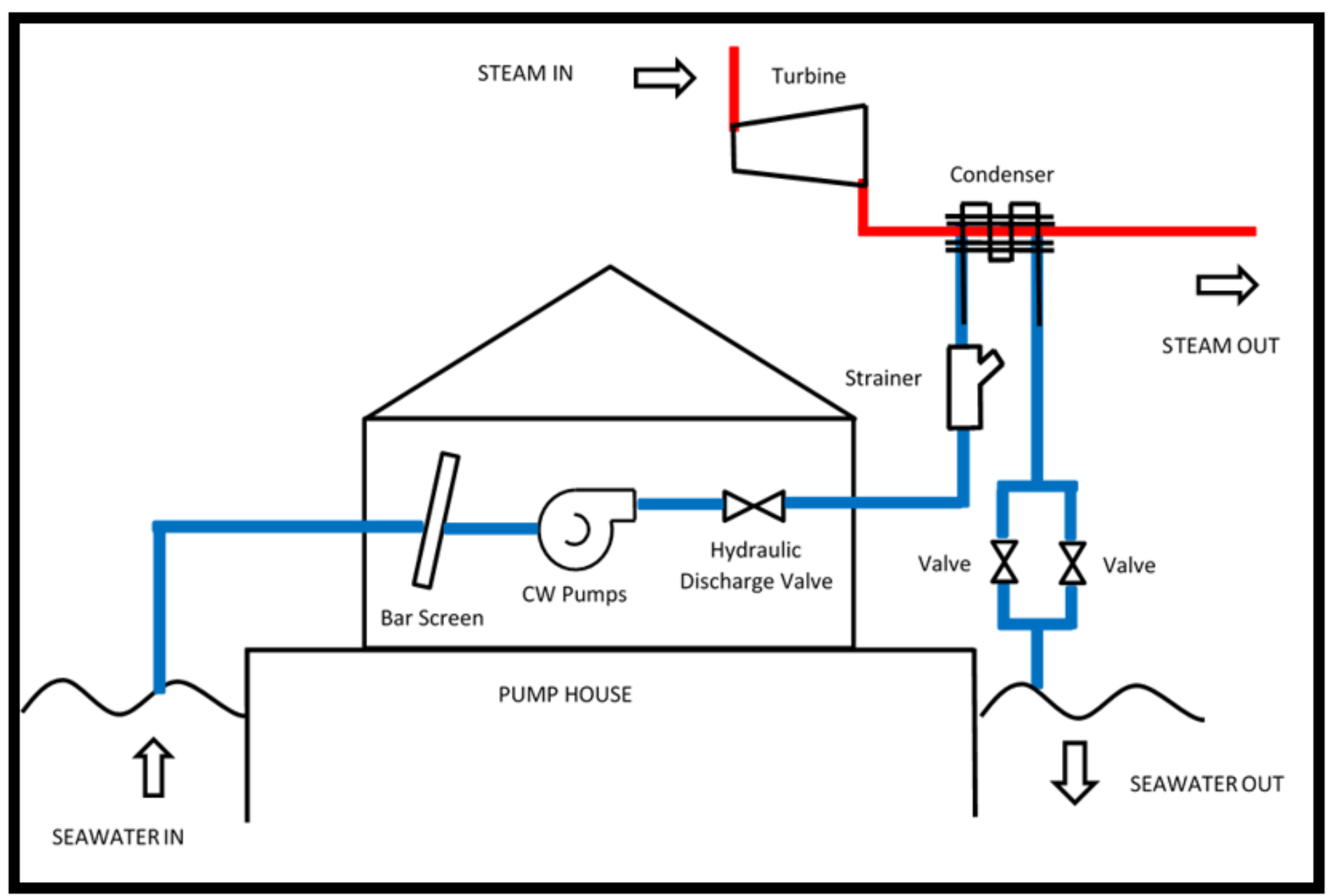

Figure 8

The circulating water (CW) system. 